\title{
Fibrocollagen-covered prosthesis for a noncircumferential segmental tracheal replacement
}

\author{
Fernando Villegas-Álvarez, MD, ${ }^{\mathrm{a}, \mathrm{b}}$ José F. González-Zamora, MD, ${ }^{\mathrm{a}}$ Angelica González-Maciel, ${ }^{\mathrm{c}}$ \\ Rosa Soriano-Rosales, ${ }^{a}$ Beatriz Pérez-Guille, ${ }^{a}$ Luis Padilla-Sánchez, MD, ${ }^{\mathrm{b}}$ Rafael Reynoso-Robles, ${ }^{\mathrm{c}}$ \\ Andrea Ramos-Morales, ${ }^{\mathrm{c}}$ Edgar Zenteno-Galindo, MD, ${ }^{\mathrm{d}}$ Armando Pérez-Torres, $\mathrm{MD},{ }^{\mathrm{e}}$ and \\ Eduardo E. Montalvo-Jave, MD, FACS ${ }^{\text {b,f }}$
}

\begin{abstract}
Objective: Fibrocollagen-covered polyester meshes can be used as possible substitutions for tracheal segments if they become integrated into the tissue without complications. The aim of this study was to assess a fibrocollagencovered polyester prosthesis to be used as a substitution for a tracheal segment.
\end{abstract}

\begin{abstract}
Methods: We performed a blind, randomized experimental assay. Adult Wistar rats were assigned to one of 2 groups. Prostheses were made by implanting polyester tubing in a group of animals to cover them with homologous collagen. They were implanted as substitutions of tracheal segments in the experimental group after creating a defect in the anterior wall of the trachea. Clinical, histomorphologic, and immunohistochemical assessments were made at 4 different time points.
\end{abstract}

\begin{abstract}
Results: The experimental group presented some respiratory distress signs during the first 7 to 10 days, such as stertors, hissing, and low motor activity. After this initial period, the symptoms subsided progressively and disappeared at the end of the first month. These respiratory symptoms caused no mortality. Initially undifferentiated monolayer cells predominated on the implant's surface, but during the last 2 months, the proportion of epithelial and ciliated cells was similar to that seen in control animals. Types I, III, and V collagen fibers were identified around the mesh. The intraluminal area of the tracheas with prostheses and prosthesis thickness were larger during the 4 months of the experiment. The increase in thickness was due to angiogenesis without evidence of fibrosis or chronic inflammation.
\end{abstract}

Conclusions: Polyester-collagen prostheses used as substitutions of tracheal segments in rats enabled the proliferation of normal respiratory epithelium and maintained tracheal function without collapse, inflammatory reaction, or secondary stenosis. (J Thorac Cardiovasc Surg 2010;139:32-7)

When permanent tracheal injuries are present, ${ }^{1-3}$ resection of the damaged area and tracheal restitution with an end-to-end anastomosis have been considered the gold standard of surgical treatment. ${ }^{4}$ Unfortunately, this is not always possible. The risk of inducing complications results from ignoring limits that should not be exceeded. ${ }^{5-7}$ Establishment of the functional lumen in the trachea is the objective of a wide array of reconstructive techniques used to date. ${ }^{8,9}$ Among them, tracheal replacement has been attempted with solid or porous prostheses. Some porous ${ }^{9}$ prostheses can be covered with collagen; collagen is a modulator of the extracellular matrix and a growth facilitator for the pseudostratified epithelium. ${ }^{10,11}$

\footnotetext{
From the Experimental Surgery ${ }^{\mathrm{a}}$ and Electron Microscopy ${ }^{\mathrm{c}}$ Laboratories, Experimental Medicine Division, Instituto Nacional de Pediatría; the Departments of Surgery, ${ }^{\mathrm{b}}$ Biochemistry, ${ }^{\mathrm{d}}$ and Cell and Tissue Biology, ${ }^{\mathrm{e}}$ School of Medicine, Universidad Nacional Autónoma de México (UNAM); and the Department of General Surgery, ${ }^{\mathrm{f}}$ Hospital General de Mexico, Mexico City, Mexico.

Received for publication May 12, 2008; revisions received Feb 10, 2009; accepted for publication April 3, 2009; available ahead of print June 24, 2009.

Address for reprints: Fernando Villegas-Álvarez, MD, Laboratorio de Cirugía Experimental, Instituto Nacional de Pediatría, Insurgentes Sur 3700-C, Col Insurgentes Cuicuilco, Delegación Coyoacán, CP 046530, México DF (E-mail: fvadiaz@ prodigy.net.mx)

$0022-5223 / \$ 36.00$

Copyright $(c) 2010$ by The American Association for Thoracic Surgery doi:10.1016/j.jtcvs.2009.04.010
}

The purpose of this work was to assess the efficacy and compatibility of a fibrocollagen-covered polyester mesh, obtained from a donor of the same species as replacement of a noncircumferential segment from the anterior side of the cervical trachea.

\section{MATERIALS AND METHODS}

The Research and Ethics Committees of the Instituto Nacional de Pediatría reviewed and approved the research protocol (June 2005). All animals were cared for according to the recommendations of the laboratory animal care guide formulated by the National Society for Medical Research, "The guide for the care and use of laboratory animals" (National Institutes of Health publication no. 85-23, revised 1985), and the Official Mexican Standard NOM-062-ZOO-1999. Male adult Wistar rats weighing 250 to $400 \mathrm{~g}$ from the animal facilities of the Research Division of the Instituto Nacional de Pediatría were used.

Forty rats were assigned a consecutive number, and the groups to be used as donors and recipients of the prosthesis, as well as the animals not undergoing operations (the control group), were assigned by a simple drawing of numbers. The donor group comprised 8 animals. The experimental and control groups were each constituted by 4 subgroups of 4 rats each, which corresponded to follow-up time (ie, 1, 2, 3, and 4 months).

\section{Implantation and Surgical Procedures}

The Eiken's technique, ${ }^{12}$ as modified by Padilla-Sánchez and colleagues, ${ }^{13}$ was used to obtain the prosthesis. A 40-denier Celanese XRZ4 polyester mesh (Celanese, Dallas, Tex) was used to cover silicon tubes (16F, 3-cm long; 


\section{Abbreviations and Acronyms \\ PBS $=$ phosphate-buffered saline \\ $\mathrm{RT}=$ room temperature}

Figure 1). The mesh-covered tubes were sterilized with $80 \%$ ethylene oxide for 24 hours and placed subcutaneously on the back of the donor animals by means of an antiseptic surgical technique. After 4 weeks, the tubes were extracted and immersed in Hank's solution at $4{ }^{\circ} \mathrm{C}$ for 6 hours and then in a $0.5 \%$ glutaraldehyde solution for 15 days. Afterward, the fibrocollagenous tubes were washed with a $0.9 \%$ saline solution for 45 minutes and cut into $1-\mathrm{cm}-$ long sections to obtain 2 fibrocollagen-covered tubes. The initial aim of this experiment was the installation of the prostheses in a single surgical time period after removing 4 cartilage rings of the cervical trachea. However, the first 5 consecutive rats presented severe dyspnea caused by the collapse of the tubular implant; these animals died or were killed during the 12 postoperative hours to spare them from further suffering. Based on this experience, we decided to implant these tubes by using a sterile technique at the left external face of the trachea and immediately underneath the second cervical cartilage, fixing them with a 6-0 Vicryl sutures (Ethicon, Inc, Somerville, NJ) in the 4 experimental groups. The other groups did not undergo operations and served as the control group.

A month later, the experimental rats underwent reoperation. After achievement of general anesthesia and using a sterile technique, through an anterior incision and blunt dissection and avoiding separation of the prosthesis from the muscular mass and the lateral face of the trachea, the anterior face of rings 2 to 6 was exposed, and at that level, a section of the tracheal surface corresponding to $30 \%$ to $40 \%$ of the anterior diameter of the trachea was resected. The produced defect was covered with the concave face of the prosthesis, eliminating excess tissue from the borders, followed by suture to the lips of the cartilage with 8 to 9 separated polypropylene stitches. After finishing the anastomosis, the muscular planes were sutured with 4-0 Vicryl sutures, and the skin was sutured with 4-0 Dermalon sutures (Covidien Ltd, Mansfield, Mass). All surgical procedures were performed with a microscope (Carl Zeiss, Essingen, Germany) and after achievement of general anesthesia with a mixture of $3 \mathrm{mg} / \mathrm{kg}$ xylazine, $35 \mathrm{mg} / \mathrm{kg}$ ketamine, and 0.05 $\mathrm{mg} / \mathrm{kg}$ atropine sulphate applied intramuscularly.

\section{Postsurgical Follow-up}

After the surgical procedure, the animals were housed in individual cages in a temperature-controlled environment and under a 12-hour/12-hour light/dark schedule. Animals were given water and food (LabDiet; Purina Mills, St Louis, Mo) ad libitum. During the immediate postoperative period, 4 sessions of micronebulization with budesonide $(250 \mathrm{mg} / 2 \mathrm{~mL})$ were applied at 12-hour intervals; enrofloxacine $(5 \mathrm{mg} / \mathrm{kg})$ and metamizol $(25 \mathrm{mg} / \mathrm{kg}$ administered intramuscularly) were administered for 3 consecutive days. On the fourth day, oxytetracycline $(10 \mathrm{mg} / \mathrm{mL}$ of water) was administered for 10 days.

\section{Clinical Assessment}

Each group was followed independently for 1, 2, 3, or 4 months, respectively, during which the presence of respiratory distress, hissing, stertors, mucosal cyanosis, hypoactivity, or death was monitored and registered every day. At the end of the observation period, rats were killed with a lethal sodium pentobarbital dose $(60 \mathrm{mg} / \mathrm{kg})$ and perfused transcardially for $3 \mathrm{~min}-$ utes with phosphate-buffered saline (PBS), $\mathrm{pH} 3.4$, and for 30 minutes with a $4 \%$ paraformaldehyde and $2.5 \%$ glurataraldehyde solution $(0.1 \mathrm{~mol} / \mathrm{L}$ PBS, pH 7.4). The tracheas were coded for blind analysis.

\section{Specimen Processing for Electron Transmission Microscopy}

The trachea was dissected by using a surgical microscope at the level of the implant, and 4 to 5 transversal segments of approximately 2-mm thick-

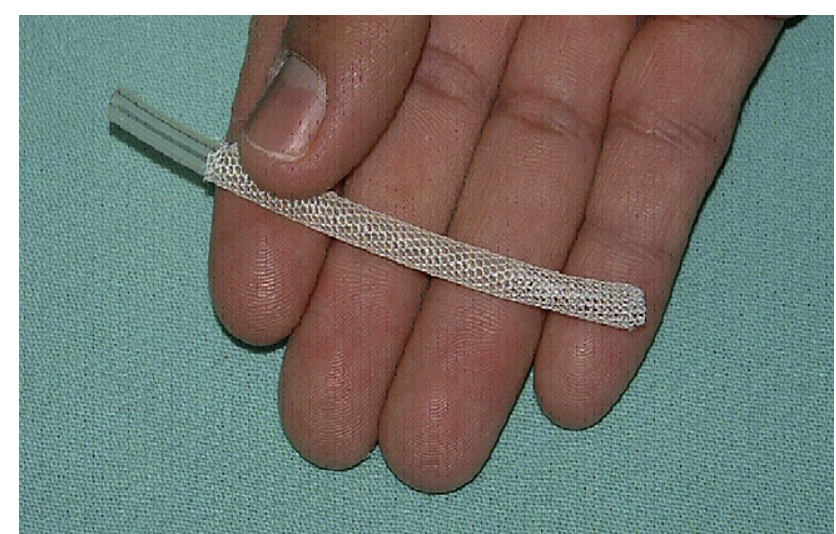

FIGURE 1. Mesh and Silastic (Dow Corning, Midland, Mich) tube before subcutaneus insertion on the backs of the donor animals.

ness were obtained. The specimens were postfixed in $2 \%$ osmium tetroxide dehydrated by means of ethanol series, and embedded in epoxy resin. Morphologic analysis was performed on semithin sections $(0.5 \mu \mathrm{m})$ stained with toluidine blue. We determined the area, total length of the implant, and the zone with or without cilia in each section. Morphometric analysis was performed with a Zeiss Axioscop-2 Plus microscope coupled to an image system analysis system, AxioVision version 4.4 (Carl Zeiss). Proximal zones to the suture stitches of the implant with the trachea and from the central part of the implant were taken from a transversal section of the trachea from each rat and group to assess the characteristics of the epithelium that developed on the implant. These samples were then re-embedded in Epon. From these samples, $60-$ to $90-\mu \mathrm{m}$ sections were obtained, placed on grids, and contrasted with uranyl acetate/lead citrate. The samples were analyzed with a Carl Zeiss EM-109 transmission electron microscope. We obtained contiguous micrographs from each sample, and approximately $12 \mu \mathrm{m}$ of each zone was reconstructed. The type and number of cells were determined on the basis of cellular morphology, as described by Cross and Mercer. ${ }^{14}$ In regions with undifferentiated cells, the number of nuclei was considered as the number of cells undergoing differentiation.

\section{Immunohistochemical Method}

Paraffin-embedded sections. The tracheas of the control and experimental rats were collected at necropsy and longitudinally sectioned at the middle line (through the prosthesis in experimental rats), and approximately $0.5-\mathrm{cm}$-long fragments were fixed with $10 \%$ buffered neutral formalin for 24 hours and then processed with paraffin embedding and sectioning. Tracheal tissue sections of 6 to $8 \mu \mathrm{m}$ in thickness were obtained, mounted on poly-L-lysine-coated slides, and stained with Masson's trichrome stain for collagen determination, mainly type I fibers; Wilder's method for reticulum fibers or type III collagen fibers; and alcian blue, $\mathrm{pH} 2.5$, for weakly acidic sulfated glycosaminoglycans and hyaluronan, according to protocols from the Armed Forces Institute of Pathology. ${ }^{15}$ The purpose was to identify the extracellular matrix components of connective tissue associated to the prosthesis.

Immunohistochemical procedures. Tracheal sections for immunohistochemistry were mounted on positive-charged slides (Superfrost plus; Shandon, Inc, Pittsburgh, Pa), deparaffinized with xylene, rehydrated with PBS, and then transferred to plastic Coplin jars containing $0.1 \mathrm{~mol} / \mathrm{L}$ citrate buffer ( $\mathrm{pH}$ 6.0) for antigen retrieval treatment, as described by Shi and associates. ${ }^{16}$ In brief, slides were heated inside the Coplin jars, which were placed in a pressure cooker for 20 minutes at the highest temperature of a hot plate (approximately $200^{\circ} \mathrm{C}$ ) and 10 minutes more at an intermediate temperature (approximately $100^{\circ} \mathrm{C}$ ). Afterward, slides were maintained in the Coplin jars for 15 minutes at room temperature (RT) and then transferred to $0.15 \mathrm{~mol} / \mathrm{L} \mathrm{PBS}$, $\mathrm{pH} 7.2$, until required. 


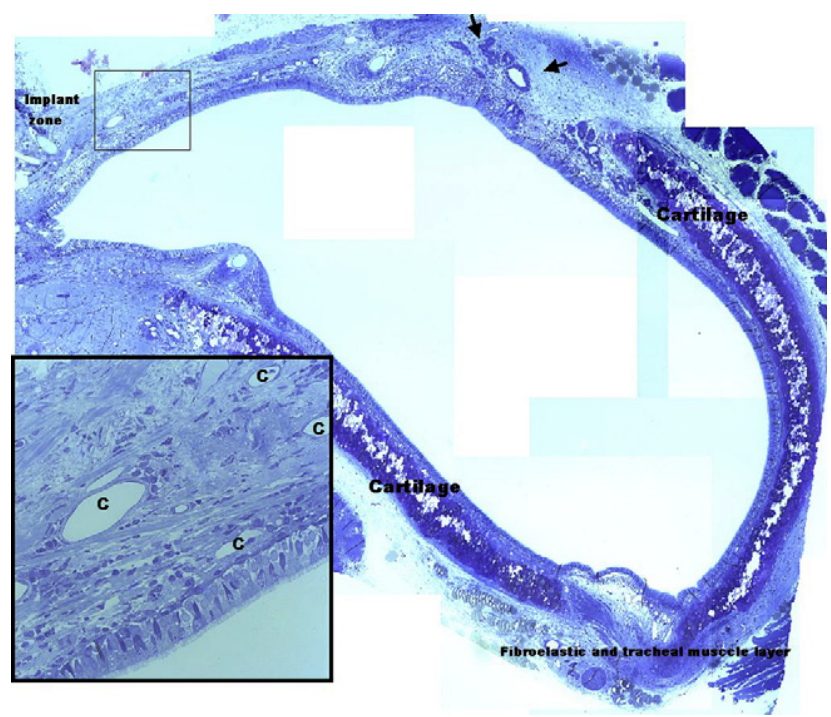

FIGURE 2. Tracheal cross-section reconstruction 4 months after implanting the prosthesis: note the deformed tracheal contour. (Toluidine blue; Original magnification $10 \times$.) The inset shows ciliated pseudostratified columnar epithelium and capillaries in the submucosa. (Original magnification 40×.) C, Capillary cartilage. Arrows indicate glands.

After antigen retrieval, endogenous peroxidase was inhibited by means of incubation in 3\% hydrogen peroxide diluted in methanol for 30 minutes at RT. Slides were then incubated for 1 hour at RT in PBS-2\% bovine serum albumin- $0.01 \%$ Triton X-100 solution to reduce nonspecific background staining, followed by overnight incubation at $4{ }^{\circ} \mathrm{C}$ with primary antibodies specific for rat fibrillar collagen type I (goat polyclonal IgG; Santa Cruz Biotechnology, Inc, Santa Cruz, Calif) or type V (mouse monoclonal antibody IgG; Chemicon International, Temecula, Calif) diluted $1: 100$ in PBS- $0.1 \%$ bovine serum albumin. After 3 washes in PBS, slides were incubated in biotinylated anti-goat or anti-mouse IgG secondary antibody for 1 hour at RT, and then an avidin-biotin-horseradish peroxidase complex was used, and the reaction was developed with $3^{\prime} 3^{\prime}$-diaminobenzidine as chromogen, according to the supplier's instructions (all reagents were from Santa Cruz Biotechnology, Inc). Negative controls were tissue sections processed as mentioned above but incubated with normal mouse or normal goat sera as primary antibodies.

\section{Statistical Analysis}

Data were tabulated and analyzed with the Shapiro-Wilks test. Normally distributed data were analyzed with the Student $t$ test for independent samples, and the Mann-Whitney $U$ test was used for nonnormally distributed data. The possible differences between the control and experimental groups at the 4 times of observation were assessed by means of ANOVA or the Kruskal-Wallis test. The SPSS version 12 statistical package (SPSS, Inc, Chicago, Ill) was used in the analyses.

\section{RESULTS}

No deaths occurred, and the implanted rats presented few cases of stertors, hissing, and low motor activity during the first 7 to 10 days. Afterward, these distress signs disappeared progressively and were nil at the end of the first month. After killing the animals, at the time of necropsy, it was found that the habitual circular morphology of the trachea had changed at the site of the prosthesis, adopting different shapes, al-

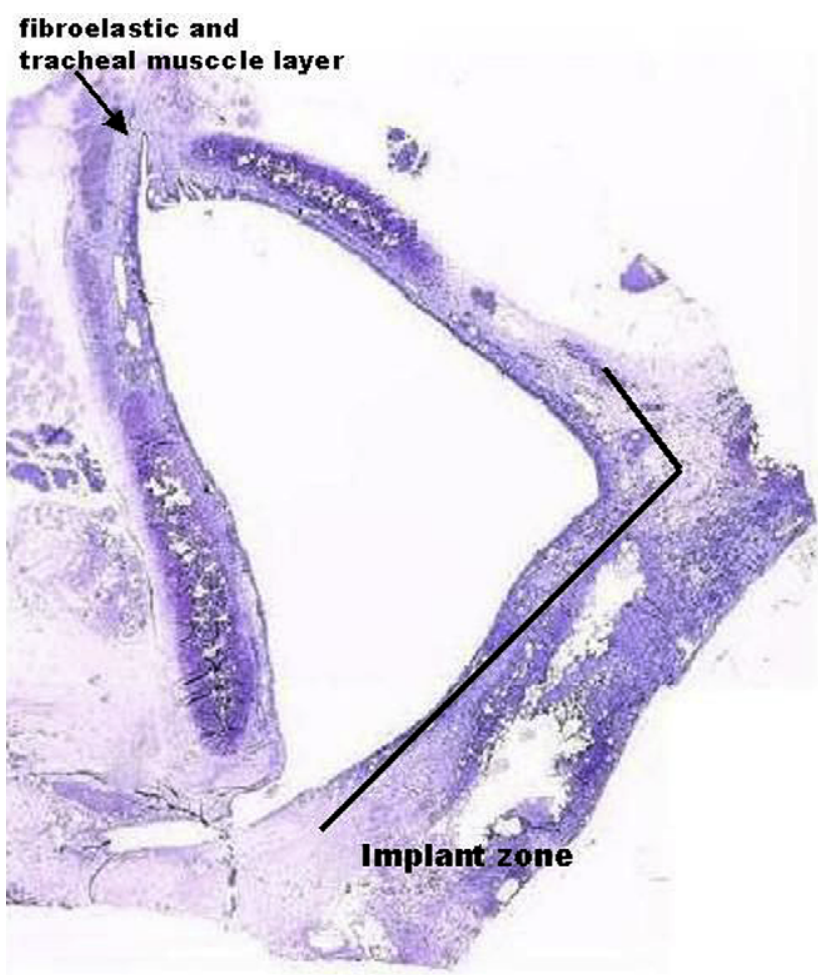

FIGURE 3. Deformation of the tracheal contour evidencing the thickness of the implant wall without affecting the lumen. The posterior wall, constituted by the noncartilaginous portion of the trachea, is contracted. (Original magnification $40 \times$.)

though 8 of the 16 presented a triangular contour with a posterior vertex (Figures 2 and 3). The intraluminal area of the trachea presented a normal distribution in both the control and experimental groups. On comparison of the intraluminal area (control vs experimental group), that of the control group was $3.71 \pm 0.21 \mathrm{~mm}^{2}$ smaller than the experimental areas at the 4 analyzed times: (1) $4.61 \pm 0.36 \mathrm{~mm}^{2}$, (2) $4.45 \pm 0.3 \mathrm{~mm}^{2}$, (3) $4.63 \pm 0.31 \mathrm{~mm}^{2}$, and (4) $4.25 \pm$ $0.35 \mathrm{~mm}^{2}(P=.05$, Student $t$ test). The thickness of the implanted wall, presenting a large dispersion and abnormal distribution at the 4 observation periods, was greater (ie, [1] $487.5 \pm 121.2 \mu \mathrm{m},[2] 721 \pm 274.8 \mu \mathrm{m},[3] 1061 \pm 275$ $\mu \mathrm{m}$, and [4] $1425 \pm 393 \mu \mathrm{m}$ ) than that of the control tracheal wall (average of $388 \pm 82 \mu \mathrm{m} ; P=.01$; Figure 4). On the surface of the implant, as well as in the control group, 4 types of cells (globular, basal, undifferentiated, and ciliated) were identified. However, during the first 2 months, undifferentiated monolayer cells predominated in the experimental group, but thereafter, the range and variety of epithelial cells were similar to those of the control group (Table 1). The amount of ciliated cells over the prosthesis oscillated from $0 \%$ in the first month to $94 \%$ and $95 \%$ in the last 2 months, respectively (Table 2 and Figure 5).

Histologic analysis showed that the increased thickness of the implant was due to the presence of abundant blood 

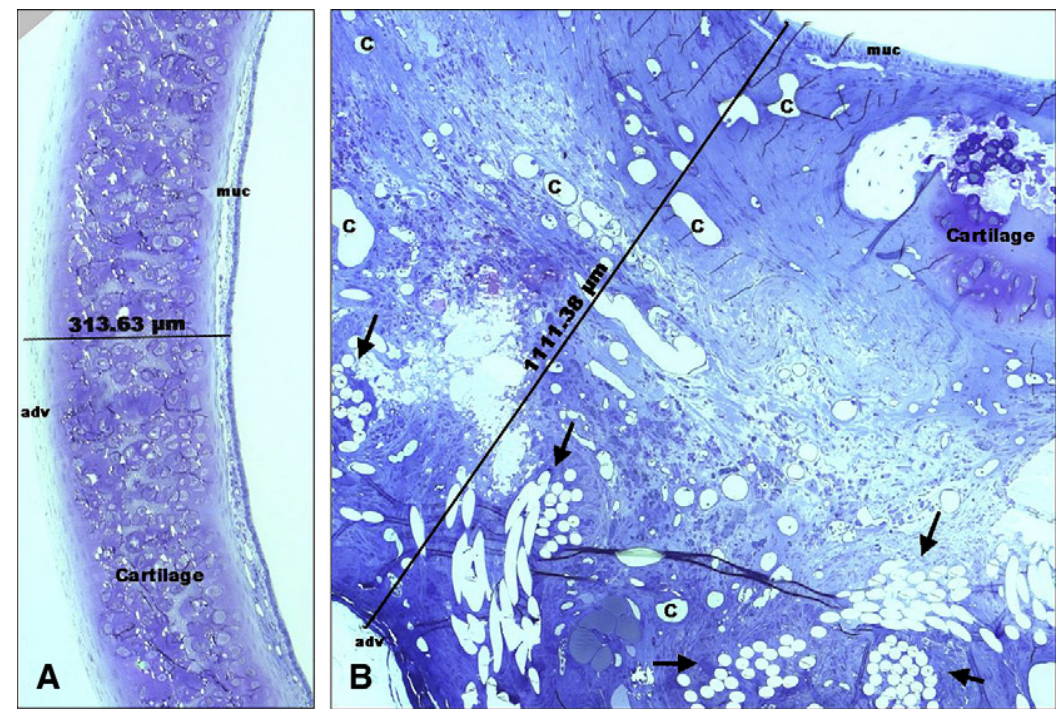

FIGURE 4. Thickness of the tracheal wall 3 months after implantation. The labeled line indicates the thickness in micrometers from the mucosa $(m u c)$ to the adventitia $(a d v)$. A, Control group; B, experimental group. The arrow shows the fibrocollagen-covered polyester mesh. We found abundant capillaries $(C)$. (Toluidine blue; original magnification $10 \times$.)

vessels, mainly arterioles, capillaries, and venules, and collagen fibers (probably types I and III) adhering to and surrounding the mesh. A scanty positive stain to glycosaminoglycans and hyaluronan covered the surface of the mesh. Immunohistochemistry corroborated that collagen types I and V were components of the implant's connective tissue matrix (Figure 6). Control results were negative.

Surprisingly, very few multinucleated giant cells and small mononuclear infiltrates were observed. However, mast cells were a frequent component of the implant (data not shown).

The prostheses from the rats that died or were initially killed were not subjected to histologic analyses because, given the short time that elapsed between surgical intervention and death, we expected to find no changes other than those derived from the surgical trauma.

\section{DISCUSSION}

The main concern after tracheal prosthetic repair is an unsuccessful tracheal function caused by inadequate structural support. De Ugarte and coworkers ${ }^{17}$ achieved the required rigidity without collapse to repair a partial circumferential

TABLE 1. Range and number of epithelial cells per field in the prostheses and their respective controls at 4 evaluated time points

\begin{tabular}{lccccc}
\hline & & \multicolumn{4}{c}{ Implant group } \\
\cline { 3 - 6 } \multicolumn{1}{c}{ Cell } & Control group & Month 1 & Month 2 & Month 3 & Month 4 \\
\hline Ciliated & $4-6$ & 0 & $1-2$ & $4-6$ & $5-6$ \\
Globose & $2-5$ & 0 & 1 & $2-3$ & 3 \\
Basal & $3-5$ & 0 & 0 & 4 & $4-5$ \\
Undifferentiated & 0 & $8-9$ & $5-7$ & 0 & 0 \\
\hline
\end{tabular}

tracheal defect by increasing the layer folds of a prosthesis made of collagen-based matrix derived from porcine small intestinal submucosa. After 4 weeks, this author observed complete epithelization and neovascularization of the tracheal patch. Nevertheless, most of the rats presented with chronic and moderate inflammation, and the long-term ability to prevent scar contracture and restenosis was not evaluated.

Collagen, particularly types I and III, induces angiogenesis, a phenomenon repeatedly observed in other studies in which collagen fiber tunnels or conduits have been used, with or without specific inducers. ${ }^{10,11,18,19}$ In this experiment we assume that angiogenesis contributed to the increase in tracheal wall thickness at the implant site, which probably increased the structural support of the collagencovered mesh, thus avoiding its collapse.

Angiogenesis is a constituent of diverse natural processes, such as placental formation and healing of wounds, ${ }^{20-22}$ or of pathologic conditions, such as ischemia, ${ }^{20}$ diabetic retinopathy, ${ }^{23}$ and is found particularly in neoplasms ${ }^{24-28}$; significant demands of oxygen and nutrients by the cells must be satisfied during these processes. In this study the use of fibrocollagen-covered prostheses from donors of the same

TABLE 2. Proportion of cilia-covered epithelium at 4 evaluation times

\begin{tabular}{lcc}
\hline $\begin{array}{c}\text { Time with } \\
\text { prosthesis (mo) }\end{array}$ & $\begin{array}{c}\text { Total implant } \\
\text { zone (mm) }\end{array}$ & $\begin{array}{c}\text { Cells covered } \\
\text { with cilia (\%) }\end{array}$ \\
\hline 1 & $2.1 \pm 0.57$ & 0 \\
2 & $2.0 \pm 0.2$ & 7 \\
3 & $2.2 \pm 0.2$ & 94 \\
4 & $1.95 \pm 0.1$ & 95 \\
\hline
\end{tabular}




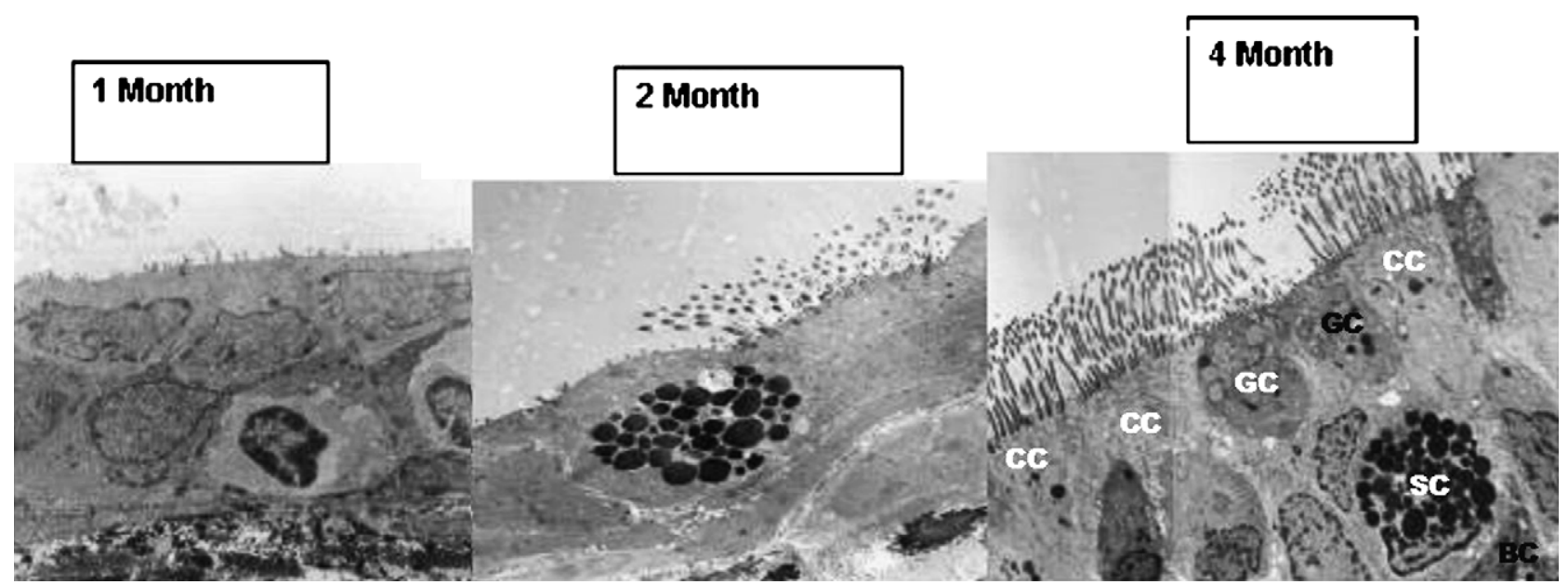

FIGURE 5. Micrograph of the mucosa at 1,2, and 4 months after implantation. On the surface of the implant, we found undifferentiated cells at 1 month, serous-like and undifferentiated cells at 2 months, and the same types of cells as found in the control group at 3 and 4 months. GC, Goblet cell; $C C$, ciliated cells; $B C$, basal cells; $S C$, some serous-like cells.

species probably improved implant acceptance, and therefore in the absence of other adverse events, such as persistent inflammation, foreign body reaction, and local infection, the observed angiogenesis could be part of a repair and tissue integration process. On the other hand, there was no scar retraction, and the intraluminal area of the trachea was larger in the experimental group than in the control group. However, this last effect was fortuitously achieved when covering the created space with the implant, which generated a larger space because of the immediate retraction of the remnant dissected cartilaginous rings, modifying the tracheal contour at that level (Figure 3).

Growth of the respiratory epithelium from the borders of the implant to the rest of its surface is another important objective. Davenport and Nettshein, ${ }^{10,11}$ in diverse experimental studies, proved that collagen is an excellent modulator of extracellular matrix and a growth facilitator for the pseudostratified epithelium.

In the present study evidence of ciliated respiratory epithelium in the whole graft surface was observed after the second month, probably because of the greater surface to be covered. It can be assumed that the lack of ciliated cells, which was particularly marked in the first few weeks, probably hindered adequate management of secretions during the postoperative period, accounting for the initially detected respiratory distress signs. However, the histologic findings from both light and electron microscopy demonstrated an excellent incorporation of the homologous fibrocollagen-covered mesh into the host tissue and the progressive regeneration of normal epithelial cells on the whole implant. The type of detected collagen, aside from stimulating angiogenesis and growth of the respiratory epithelium, probably eased the production, storage, and release of chemical mediators, cytokines, growth factors, and others as inducers of tissue repair.
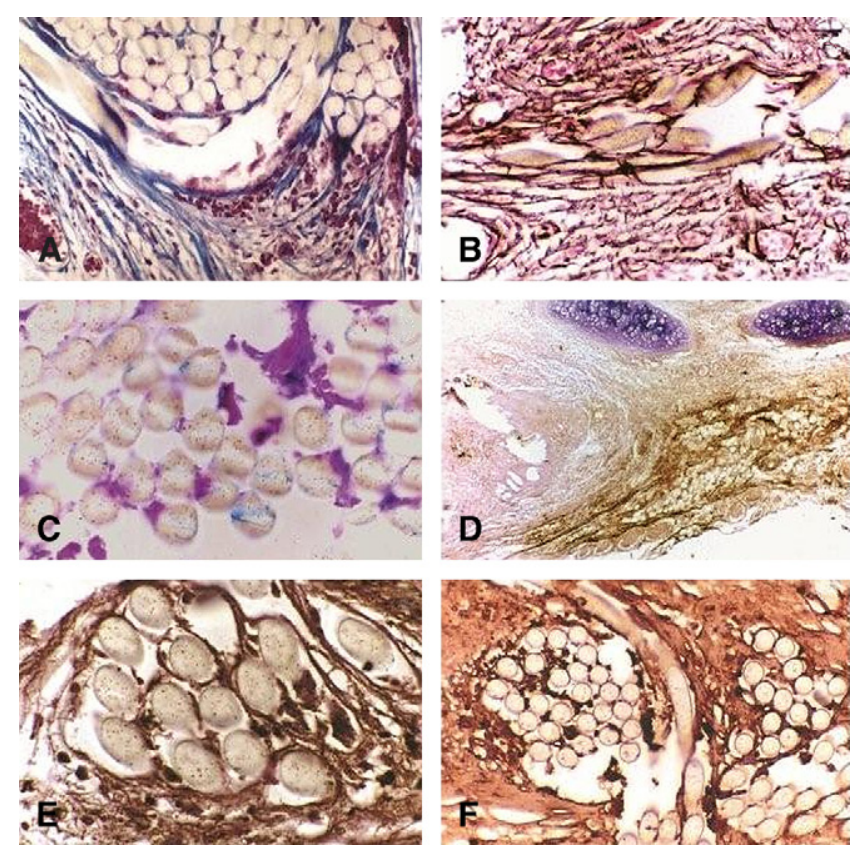

FIGURE 6. Histology and immunohistochemistry of a fibrocollagen-covered prosthesis for segmental tracheal replacement in a rat. Collagen staining with either Masson's trichrome stain (A) or Wilder's method (B) demonstrated the presence of a layer of collagen (blue and pink fibers, respectively) already interwoven into the mesh and surrounding it. Moreover, black fibers corresponding to reticular fibers or type III collagen (B) were a prominent component of the connective tissue matrix of the mesh. Hyaluronan and glycosaminoglycans were also observed as a blue stain over the mesh (C). Immunohistochemistry for type I (D and E) and type $\mathrm{V}(\mathrm{F})$ collagen (dark brown stain) confirmed the histologic findings of collagen staining. Note the presence of numerous blood vessels near the mesh (A). (Original magnification: A, B, and F, 100×; C and E, $200 \times ; \mathrm{D}, 40 \times$.) 
Tissue repair with a biocompatible substitute structure is an old desire; however, this must ideally be free of leaks, be noncollapsible, be tolerated by the host, show minimal or no inflammatory reaction, and be impermeable to fibroblasts and bacterial invasion of the lumen, aside from being able to cover the whole respiratory epithelium. ${ }^{29}$

Some of these objectives were met in this experiment. However, to avoid the collapse of the implant, it was necessary to reduce the size and to perform an extrasurgical procedure to attach and stabilize it to the adjacent tracheal muscles to facilitate local support. Other challenges for the future will be to assess this type of prosthesis with autologous collagen, particularly in young subjects, who we assume will accept it more readily. To be able to apply this technique as a therapeutic resource in pediatric patients, not only is immediate correction of the defect that originated the tissue substitution warranted, but also the long-term effects during tracheal growth along age must be addressed.

One of the most important clinical reports done recently is the use of a bioprosthesis of Marlex mesh covered with a porcine collagen sponge, autologous blood, and additional support of polypropylene rings, which was applied successfully for the first time in a 78-year-old woman. The implant was not only well tolerated and integrated into the surrounding tissues, but it also remained functional and without complications for 2 years after implantation. ${ }^{30}$ Reports such as these enable us to assume that substitution of tracheal defects with meshes made from diverse medical-use materials and covered with collagen will, in the near future, be an additional alternative to the conventional or very sophisticated tracheobronchial repair techniques.

\section{CONCLUSIONS}

The polyester collagen prosthesis, used as a substitution for a tracheal segment in rats, allowed normal functioning without collapse and normal epithelization without chronic inflammatory reaction or secondary tracheal stenosis. The scientific, social, and economic effects of the use of fibrocollagen-covered prostheses are very promising, but research must be continued to improve these implants, including models to be tested during specific growth stages, because this can be a limiting factor.

\section{References}

1. Carden KA, Boiselle PM, Waltz DA, Ernst A. Tracheomalasia and tracheobroncomalasia in children and adults: an in-depth review. Chest. 2005;127:984-1005.

2. Sue RD, Susanto I. Long-term complications of artificial airways. Clin Chest Med. 2003;24:457-71.

3. Gomes CAM, Fernandez JC, Troster EJ. Possible risk factors associated with moderate or severe airway injuries in children who underwent endotracheal intubations. Pediatr Crit Care Med. 2004;5:364-8.
4. Healy GB, Shuster SR, Jonas RA, McGill TJI. Correction of segmental tracheal stenosis in children. Ann Otol Rhinol Laryngol. 1988;97:444-7.

5. Mulliken JB, Grillo HC. The limits of tracheal resection with primary anastomosis: further anastomotical studies in man. J Thorac Cardiovasc Surg. 1968;55: 418-21.

6. Myssiorek D. Recurrent laryngeal nerve paralysis: anatomy and etiology. Otolaryngol Clin North Am. 2004;37:25-44.

7. Islam S, Masiakos PT, Doody DP, et al. Tracheal resection and reanastomosis in the neonatal period. J Pediatr Surg. 2001;36:1262-5.

8. Kucera KA, Doss AE, Dunn SS, Clemson LA, Zwischenberger JB. Tracheal replacement: part 1. ASAIO J. 2007;53:497-505.

9. Doss AE, Dunn SS, Kucera KA, Clemson LA, Zwischenberger JB. Tracheal replacement: part 2. ASAIO J. 2007;53:631-9.

10. Davenport EA, Nettsheim P. Type I collagen gel modulates extracellular matrix synthesis and deposition by tracheal epithelial cells. Exp Cell Res. 1996;223: 155-62.

11. Davenport EA, Nettesheim P. Regulation of mucociliary differentiation of rat tracheal epithelial cells by type I collagen gel substratum. Am J Respir Cell Mol Biol. 1996;14:19-26.

12. Eiken O, Norden G. Bridging. Small artery defects in the dog with in situ preformed autologous connective tissue tubes. Acta Chir Scand. 1961;121: 90-102.

13. Padilla-Sánchez L, Carrillo-Soto IA, García-Garduño MV. Valdes Gonzalez R Angiogenesis inducida por túneles de fibrocolagenos. Cir Gral. 1996;10:123-8.

14. Cross PC, Mercer KL. Cell and tissue ultrastructure. New York: WH Freeman and Company; 1993.

15. Prophet EB, Mills B, Arrigton JB, Sobin LH. Laboratory methods in histotechnology. Washington, DC: Armed Forces Institute of Pathology; 1992:127-46; 149-70.

16. Shi S-R, Chaiwn B, Young L, Cote RI, Taylor CR. Antigene retrieval technique utilizing citrate buffer or urea solution for immunohistochemical demonstration of androgen receptor in formalin-fixed paraffin sections. J Histochecytochem. 1993; 41:1599-604.

17. De Ugarte DA, Puapong D, Roostaeian JBA, et al. Surgisis match tracheoplasty in a rodent model for tracheal stenosis. J Surg Res. 2003;112:65-9.

18. Padilla SL, Figueroa SB, de León D, et al. Angiogénesis inducida por colágena polivilpirrilidona y heparina en músculo isquémico. Cir Cir. 1999;67:59-65.

19. Padilla L, Krotzsh E, Schalch P, et al. Administration of bone marrow cells into surgically induced fibrocollagenous tunnels induces angiogenesis in ischemic rat hindlimb model. Microsurgery. 2003;23:568-74.

20. Padilla L, Krotzsch E, De la Garza A, et al. Bone marrow mononuclear cells stimulate angiogenesis when transplanted into surgically induced fibrocollagenous tunnels: results with a canine ischemic hindlimb model. Microsurgery. 2007; 27:91-7.

21. Losordo DW, Isner JM. Estrogen and angiogenesis. Arterioscler Thromb Vasc Biol. 2001;21:6-12.

22. Demir R, Kayisli UA, Cayli S, Huppertz B. Sequential steps during vasculogenesis and angiogenesis in the very early human placenta. Placenta. 2006;27:535-9.

23. Liekens S, Clercq ED, Neyts J. Angiogenesis: regulators and clinical applications. Biochem Pharmacol. 2001;61:253-70.

24. Ferrara N, Alitalo K. Clinical applications of angiogenic growth factors and their inhibitors. Nat Med. 1999;5:1359-64.

25. Gasparini G. The rationale and future potential of angiogenesis inhibitors in neoplasia. Drugs. 1999;58:17-38.

26. Kaklamanis L, Kakolyris S, Koukourakis M, et al. From hyperplasia to neoplasia and invasion: angiogenesis in the colorectal adenoma-carcinoma model. Adv Exp Med Biol. 2000;476:249-66.

27. Carmeliet P, Jain RK. Angiogenesis in cancer and other diseases. Nature. 2000 407:249-57.

28. Xu L, Cochran DM, Tong RT, Winkler F, et al. Placenta growth factor and metastasis by depleting vascular endothelial growth factor homodimers in orthotopic mouse models. Cancer Res. 2006;66:3971-7.

29. Neville WE, Bolanowski PJP, Soltanzadeh H. Prosthetic reconstruction of trachea and carina. J Thorac Cardiovasc Surg. 1976;72:525-38.

30. Omori K, Nakamura, Kanemaru S, et al. Regenerative medicine of trachea: the first human case. Ann Otol Rhinol Laryngol. 2005;114:429-33. 